\title{
Off-line and on-line optical monitoring of microalgal growth
}

\author{
Hugo-Enrique Lazcano-Hernandez ${ }^{1}$, Gabriela Aguilar ${ }^{2}$, Gabriela Dzul ${ }^{2}$, Rodrigo Patiño ${ }^{\text {Corresp., }}{ }^{2}$, Javier Arellano- \\ Verdejo ${ }^{\text {Corresp. } 3}$ \\ ${ }^{1}$ Cátedras CONACYT-El Colegio de la Frontera Sur, Chetumal, Quintana Roo, México \\ 2 Departamento de Física Aplicada, Cinvestav Unidad Mérida, Mérida, Yucatán, México \\ 3 Estación para la Recepción de Información Satelital ERIS-Chetumal, El Colegio de la Frontera Sur, Chetumal, Quintana Roo, México \\ Corresponding Authors: Rodrigo Patiño, Javier Arellano-Verdejo \\ Email address: rodrigo.patino@cinvestav.mx, javier.arellano@mail.ecosur.mx
}

The growth of Chlamydomonas reinhardtii microalgae cultures was successfully monitored, using classic off-line optical techniques (optical density and fluorescence) and on-line analysis of digital images. In this study, it is shown that the chlorophyll fluorescence ratio $\mathrm{F}_{685} / \mathrm{F}_{740}$ has a linear correlation with the logarithmic concentration of microalgae.

Moreover, with digital images, the biomass concentration was correlated with the luminosity of the images through an exponential equation and the length of penetration of a superluminescent blue beam $(\lambda=440 \mathrm{~nm}$ ) through an inversely proportional function. The outcomes of this study are useful to monitor both research and industrial microalgae cultures. 


\title{
Off-line and on-line optical monitoring of microalgal growth
}

\author{
Hugo E. Lazcano-Hernandez ${ }^{1}$, Gabriela Aguilar², Gabriela Dzul' \\ Rodrigo Patiño ${ }^{2}$, and Javier Arellano-Verdejo ${ }^{3}$
${ }^{1}$ Cátedras CONACYT-EI Colegio de la Frontera Sur, Chetumal, Quintana Roo, México ${ }^{2}$ Departamento de Física Aplicada, Cinvestav Unidad Mérida, Mérida, Yucatán, México ${ }^{3}$ El Colegio de la Frontera Sur, Estación para la Recepción de Información Satelital ERIS-Chetumal, Chetumal, Quintana Roo, México.

9 Corresponding author:

10 Javier Arellano-Verdejo, Rodrigo Patiño

11 Email address: javier.arellano@mail.ecosur.mx, rodrigo.patino@cinvestav.mx

\begin{abstract}
The growth of Chlamydomonas reinhardtii microalgae cultures was successfully monitored using classic off-line optical techniques (optical density and fluorescence) and on-line analysis of digital images. In this study, we found that the chlorophyll fluorescence ratio $F_{685} / F_{740}$ has a linear correlation with the logarithmic concentration of microalgae. By using digital images, the biomass concentration correlated with the luminosity of the images through an exponential equation and the length of penetration of a super luminescent blue beam $(\lambda=440 \mathrm{~nm})$ through an inversely proportional function. The outcomes of this study are useful for both research and industrial microalgae cultures.
\end{abstract}

\section{INTRODUCTION}

Photosynthesis is a biophotonic mechanism by which green plants, cyanobacteria and algae produce their own food by transforming a fraction of solar energy to biochemical energy. This is the foundation of life on Earth. Photosynthesis occurs in the chloroplasts, which are cell organelles that contain photosynthetic pigments (chlorophyll a, chlorophyll b, carotenoids, etc.). They absorb light and use it to drive photosynthetic light reactions and associated electron transport reactions to reduce $\mathrm{CO}_{2}$ and oxidize $\mathrm{H}_{2} \mathrm{O}$ in the Calvin cycle (Allen, 1992). The net result of photosynthesis is the production of carbohydrates and the release of molecular oxygen to the atmosphere. Environmental factors such as temperature, irradiance, humidity and salinity are known to affect photosynthesis (Rym, 2012).

Microalgae cultivation has been widely studied due to its potential as a source of food, biofuel, and various bioactive compounds (Pulz and Gross, 2004; Spolaore et al., 2006; Mata et al., 2010; Niccolai et al., 2019). These are useful for important processes such as residual water cleaning, $\mathrm{CO}_{2}$ capture, and $\mathrm{H}_{2}$ synthesis (Wang et al., 2008; Abdel-Raouf et al., 2012; Show et al., 2019). All these are valuable contributors to the balance and growth of human activity on a global scale (Gupta et al., 2015). Havlik and colleagues compiled a wide review of on-line and off-line technologies to monitor physicochemical and biological parameters of microalgae (Havlik et al., 2016). There are also several photobioreactor models to predict growth (Pulz, 2001; Carvalho et al., 2006; Xu et al., 2009). However, actual measurements are required to monitor and optimize the algae growth process. Disadvantages of sampling include the potential for contaminating the culture, disturbing the algae's physiological state or modifying the volume of the medium. Another challenge for real-time measurements is the wide range of concentrations encountered in microalgal cultures. The concentration routinely increases by up to three orders of magnitude, preventing direct measurement by most analytical methods (Antal et al., 2019). Therefore, it is currently a challenge to implement non-invasive real-time methodologies for monitoring microalgal cultivation conditions and photosynthetic parameters (Antal et al., 2019).

Chlorophyll $(\mathrm{Chl})$ molecules are organized into two different light systems called Photosystem I (PSI) and Photosystem II (PSII). Both are spatially separated in the chloroplasts' thykaloid membranes (Breijo 
et al., 2006). Every photosystem contains an antenna light-harvesting complex (LHC) and central $C h l$ molecules. The photosystems differ from each other in their proportions of Chl a, Chl b, the characteristics of their reaction centers, and the electron carriers involved in their processes. In PSI, the reactive center is called P700 and is formed by two Chl a molecules that are attached to each other. PSII also contains a reactive center called $P 680$ which is formed by two attached $C h l$ a molecules. The nomenclature is associated with the maximum wavelength $(\lambda)$ absorption of both $P S I$ and $P S I I: \lambda=700 \mathrm{~nm}$ and $\lambda=680$ $\mathrm{nm}$, respectively (Gouveia-Neto et al., 2011). The maximum fluorescence wavelength may vary according to the origin and kind of $\mathrm{Chl}$, the culture medium, the environmental conditions, and the measurement equipment. For example, at room temperature, Chl $a$ fluorescence around $\lambda=685 \mathrm{~nm}$ is largely emitted by $P S I I$ antenna, and fluorescence around $\lambda=740 \mathrm{~nm}$ is emitted by PSI antenna (Krause and Weis, 1984; Roháček et al., 2008; Gouveia-Neto et al., 2011). In the fluorescence emission spectra of healthy, suspension-diluted thylakoid membranes or isolated chloroplasts, a sharp peak around $\lambda=685 \mathrm{~nm}$ with a broad shoulder at about $\lambda=740 \mathrm{~nm}$ has been observed (Krause and Weis, 1991). Although isolated Chl $b$ dissolved in an organic solvent exhibits fluorescence, this does not happen with in vivo cultures because the excitation energy is transferred completely to Chl a (Gouveia-Neto et al., 2011).

The main function of the $L H C$ is to transfer excitation energy to the photosynthetic reaction centers, where photochemical reactions take place. However, a part of the absorbed light energy is dissipated as heat or emitted as fluorescence (Misra et al., 2012). In other words, to return to the ground state, the excited Chl molecule undergoes one of three processes including: (i) driving photochemical reactions (photosynthesis), (ii) dissipating as heat (thermal de-excitation), or (iii) being re-emitted as light (fluorescence). These processes occur in competition so that any increase in the efficiency of one will result in a decrease in the yield of the other two. Chl fluorescence is an intrinsic signal emitted by plants, algae and cyanobacteria that can be employed to monitor changes in their physiological state, such as in the photosynthetic apparatus, developmental processes, state of health, stress events and stress tolerance. It can also be used to detect diseases or nutrient deficiency (Gouveia-Neto et al., 2011; Hák et al., 1990). Hence, by measuring the yield of $\mathrm{Chl}$ fluorescence, information about changes in the efficiency of photosynthesis and heat dissipation can be obtained (Maxwell and Johnson, 2000; Krause and Weis, 1984). Therefore, the simultaneous measuring of $C h l$ fluorescence at $\lambda=685 \mathrm{~nm}\left(F_{685}\right)$ and $\lambda=740 \mathrm{~nm}\left(F_{740}\right)$ allows for an approximate non-destructive determination of $C h l$ content using the $C h l$ ratio $\left(F_{685} / F_{740}\right)$ (Hák et al., 1990).

Depending on the type of study and the suitability of the photosynthetic system, different fluorescence techniques have been used (Mauzerall, 1972; Olson et al., 1996; Kolber et al., 1998; Gorbunov and Falkowski, 2004; Johnson, 2004; Chekalyuk and Hafez, 2008). Presently, two Chl fluorescence approaches are successfully used to monitor photosynthetic efficiency in microalgae mass cultures: rapid fluorescence induction and the saturation-pulse method (Masojídek et al., 2011). Regarding outdoor algae cultures, specific fluorimeters have been used. The pulse amplitude modulation $(P A M)$ fluorimeter provides rapid light responses curves of PSII. The dual PAM fluorimeter estimates PSI and PSII yields, and the Induction Kinetics fluorimeter measures fluorescence induction curves (Sukenik et al., 2009; Kromkamp et al., 2009; Masojídek et al., 2010). On the other hand, the dry weight of microalgae correlated with the optical density (OD) of the algae cultures (del Campo et al., 2014). Limitations of the OD technique, as well as its applications to other species and another culture medium, were discussed by Griffiths and co-workers (Griffiths et al., 2011). However, real-time non-invasive methodologies are still needed to monitor microalgae culture growth conditions. Therefore, two room temperature fluorescence methods to measure Chlamydomonas reinhardtii ( $C$. reinhardtii) culture growth are proposed in this study. The first is an analytic off-line optical technique and the second involves on-line digital image analysis. The study of $C$. reinhardtii here is relevant since it is considered one of the most promising eukaryotic $\mathrm{H}_{2}$ producers (Torzillo et al., 2015). It is possible to apply the proposed methods to other species but results will vary according to specific strain features, the culture medium, PBR geometry, light sources, and temperature. However, the methods are simple and easy to implement and assess.

This project aimed at identifying alternative optical techniques for monitoring microalgal growth. Our hypotheses were that $C$. reinhardtii cell culture concentration correlates with (1) the off-line $C h l$ fluorescence ratio $F_{685} / F_{740}$ and (2) the on-line image culture color, and (3) the on-line image culture fluorescence. 


\section{MATERIALS AND METHODS}

\subsection{Microalgae cultures}

C. reinhardtii (CC-124) microalgae were purchased from the Chlamydomonas Resource Center (USA) and were grown photoautotrophically in a Sueoka medium (Sueoka, 1960). Growth conditions included continuous air bubbling $(1 V V M=1 L$ - air $/ \mathrm{min} / L$ - medium $)$ under controlled room temperature conditions $(298 \pm 2) \mathrm{K}$. Experiments were performed in two series. The first series was conducted for the off-line optical density and fluorimetry measurements (Experiments A and B). The second series was conducted for the on-line techniques that used digital images (Experiments 1 to 5). A portable spectrometer (StellarNet, EPP2000) was used to measure the optical density, fluorescence and color of the microalgae cultures, with a detection range between 200 and $850 \mathrm{~nm}$. Spectrawiz software (StellarNet, OS V5.0 (C2011) was used for spectrometer measurements. In order to monitor cell growth, OD measurements at $\lambda=640 \mathrm{~nm}$ were taken. Each measurement was repeated three times for every sample.

For the off-line experiments, three $1-L$ Roux culture bottles were used, each with $0.9 L$ of algae culture and continuous air bubbling $(1 \mathrm{~L} / \mathrm{min})$, under controlled room temperature conditions (Figure 1a). The cultures were illuminated continuously with two fluorescent lamps (Philips, F20T12/D20W). To assess the microalgae growth kinetics, three samples of $3 \mathrm{~mL}$ each were taken from every bottle every 12 hours for five days. Additionally, a final control measurement was taken on the seventh day. Triplicate $\mathrm{OD}$ and fluorescence measurements were taken for every sample. Two experiments were performed with the only difference between them being the initial microalgae concentration $\left(x_{0}\right)$. These were labeled Experiment $A\left(x_{0}=34 \pm 2 \mathrm{mg} / L\right)$, and Experiment $B\left(x_{0}=42 \pm 2 \mathrm{mg} / L\right)$. In summary, a total of 270 measurements were made for every off-line experiment and the number of measurements provided statistical significance to this study.

For the on-line experiments, a $3-L$ column photo-bio-reactor $(P B R)$ was used (Figure 1b). A transparent acrylic tube with a thickness of $25 \mathrm{~mm}$ and an inner diameter of $95 \mathrm{~mm}$ was used to build the $95-\mathrm{cm}$ length $P B R$. Four fluorescent lamps (Tecno Lite, $T 46500 \mathrm{~K} 20 \mathrm{~W}$ ) were used to illuminate the center of the column with around $100 \mu$ mol photons $m^{-2} s^{-1}$. To avoid external light, the PBR was placed inside a dark cabin. Five experiments were performed, during the five days of sampling. These included three experiments with continuous illumination (Experiments 1,2, and 3), and two experiments with 12-h light/12-h dark cycles (Experiments 4 and 5). Both off-line samples and digital images were taken every 6 or $12 \mathrm{~h}$. A webcam (Logitech, Carl Zeiss Tessar HD 1080 p) connected to a PC was used to capture the PBR digital images. For the color analysis, the images were captured with two fluorescent lamps located behind the $P B R$. These lamps were turned on while the other two lamps remained turned off. The fluorescent images, were captured in darkness and only the culture was illuminated with a blue beam from a super luminescent diode (maximal wavelength of $440 \mathrm{~nm}$ ) and a light filter (LSR-GARD $A R G O N$, model 2204) with a protection range within $190-520 \mathrm{~nm}$. In order to measure fluorescence after a dark-adapted period, at least 15 min of darkness was provided before fluorescent stimulation. The images were analyzed in a LCD screen with an Integrating cube (StellarNet, IC2) and portable spectrometer. The International Commission on Illumination (CIELAB) scale was used for color measurements. To achieve a homogeneous representation of the color of every image, five measurements were taken from different regions of the PBR.

\subsection{Fluorescence cabin}

The experimental system to measure fluorescence was composed of two main parts: a fluorescence cabin and the portable spectrometer. Based on the spectrometer, the fluorescence cabin was designed, manufactured, and coupled to the system using an optical fiber (StellarNet, F400). Through this fiber, light was guided from the sample cuvette to the spectrometer detector. The fluorescence cabin configuration is shown in Figure 2a. The main components are a dark cabin, a cylindrical (14 mm i.d.) glass sample cuvette (4 mL), six light emission diodes (LED), feed and switching electronic circuits, an AC/DC electric current converter (output $5.4 \mathrm{~V}$ ), and a multi-modal optical fiber connector. The dark cabin is a space where light does not come in from external sources. Inside the dark cabin, a base was used to fix the cuvette in a normal position (at 90 degrees) relative to the floor. As exciting radiation, six LEDs (Steren, Ultra Blue) were placed parallel to the floor, three to the right side of the sample cuvette and three to the left. This configuration ensured homogeneous illumination conditions. In Figure $2 b$, the spectrum of the six LEDs is shown, with a maximum wavelength emission at around $\lambda=464 \mathrm{~nm}$, luminosity of $7 \mathrm{~cd}$ and $400 \mathrm{~mW}$ as maximum power. 


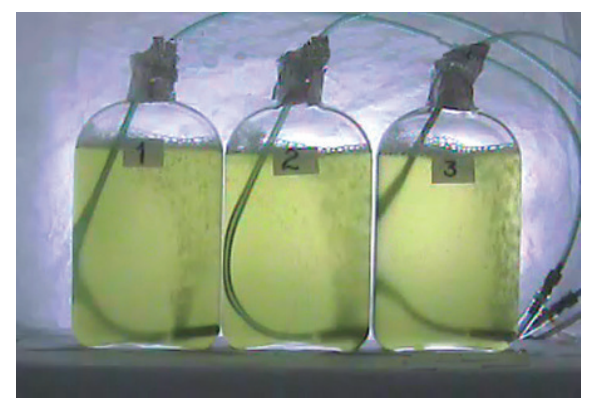

(a)

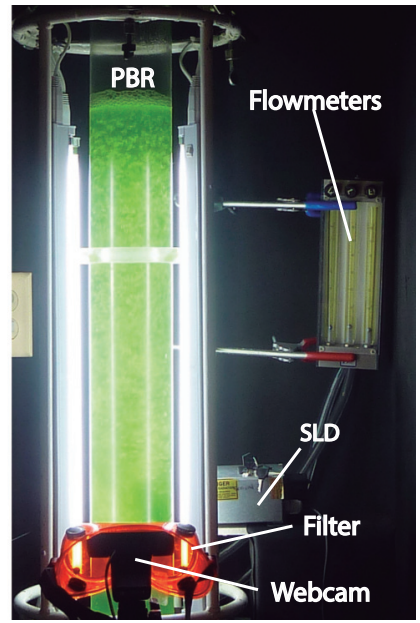

(b)

Figure 1. (a) The three Roux bottles for the off-line experiments (A and B), and (b) the column photo-bio-reactor (PBR) for the on-line experiments (1-5), Super Luminescent Diode (SLD); see Materials and Methods.

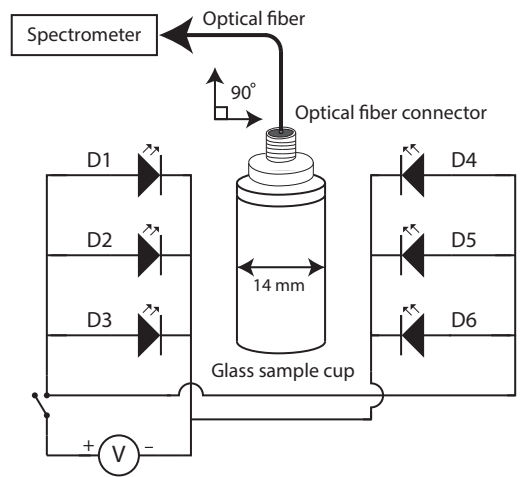

(a)

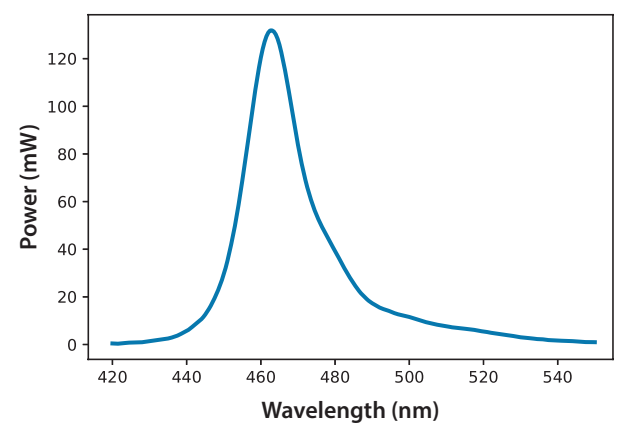

(b)

Figure 2. (a) Fluorescence cabin diagram, with optical fiber connector and glass sample cuvette; six Ultra Blue LEDs were installed as exciting source. (b) Spectrum of the exciting radiation source (Ultra Blue LEDs, $\lambda$ max $=464 \mathrm{~nm}$ ).

To improve the quality of fluorescence measurement, the optical fiber was positioned on top of the glass sample cuvette, at $90^{\circ}$ relative to the LEDs' radiation. Fluorescence traditionally is measured through the sample cuvette wall, but our fluorescence measurements were taken at the uncovered top of the cuvette, thus reducing losses from reflection and refraction in the interface of the cuvette. The cuvette used for the samples was a 4-mL glass cylinder, but with this fluorescence system, the cuvette's geometry is not important. Light information was processed and digitalized using the spectrometer SpectraWiz software. Before measurements, the reference blank was defined by setting the spectrometer with the Sueoka medium, then the fluorescence measurement was performed on the microalgae sample. The direct fluorescence of $C$. reinhardtii culture samples at room temperature was measured successfully for seven days with this experimental setup. The ratio of fluorescence intensity between $\lambda=685$ and $\lambda=740 \mathrm{~nm}$ was calculated.

\subsection{The $F_{685} / F_{740}$ fluorescence ratio}

At low $C h l$ concentrations, fluorescence emissions increase with increasing amounts of $C h l$. At higher concentrations, the increase of fluorescence with the increment of $\mathrm{Chl}$ is mainly detected around $740 \mathrm{~nm}$. For in vivo cultures, fluorescence emission at $740 \mathrm{~nm}$ is favored and fluorescence emission at $685 \mathrm{~nm}$ is 
not favored. This is due to the following factors: (i) the re-absorption of photons from the fluorescence emitted by neighboring molecules, (ii) light interference between the short $(685 \mathrm{~nm})$ and long wavelengths (740 nm), and (iii) the increment of $C h l$ (the new $C h l$ molecules preferentially absorb energy at $685 \mathrm{~nm}$ ) (Gouveia-Neto et al., 2011).

There is a good inverse correlation between photochemistry and $C h l$ fluorescence. The ratio of fluorescence intensity between maximal wavelengths $\left(F_{685} / F_{740}\right)$ is influenced by photosynthetic activity. In mature microalgae cultures, the chloroplast structure, $\mathrm{CO}_{2}$ uptake rate, carbon metabolism, etc., are better than in younger cells. Higher $F_{685} / F_{740}$ values signal young cultures or cultures with a photosynthetic apparatus that isn't developing. Low values of this rate indicate mature cultures with a fully developed photosynthetic apparatus. In other words, a decrement in $F_{685} / F_{740}$ values is indicative of increased photosynthetic activity. Measured by induction fluorescence, $F_{685} / F_{740}$ exhibits a curvilinear relationship with cell concentration $(x)$. This relationship is successfully expressed in equation 1 , where $c$ and $d$ are constants (Hák et al., 1990):

$$
\frac{F_{685}}{F_{740}}=c x^{-d}
$$

This technique has been applied to all kinds of leaves, chloroplast suspensions and acetone extracts of photosynthetic pigments. Our study demonstrated that this technique is also applicable to microalgae cultures.

\section{RESULTS AND DISCUSSION}

\subsection{Microbial growth}

It has been established that the Gompertz model represents $C$. reinhardtii growth better than the classical Monod model (del Campo et al., 2014). Actually, the Monod and Gompertz models can be seen as particular cases of a more universal growth model (Castorina et al., 2006). For the experiments in the Roux bottles (A and B), as well as in the $P B R(1-5)$, the Gompertz specific growth rate is reported in Table 1. For the $P B R$ experiments, it was possible to observe that the Gompertz model is best fitted when the light regime is continuous (see $R^{2}$ for Experiments 1-3) than when light/dark cycles are performed (Experiments 4-5). Moreover, the specific growth rate is favored when the $P B R$ is used. This stands in contrast to the cultures in the Roux bottles.

\begin{tabular}{l|c|c|c}
\hline Reactor & Experiments & $\mu\left(\right.$ day $\left.^{-1}\right)$ & $R^{2}$ \\
\hline \multirow{4}{*}{ Roux bottles } & AI & 0.5472 & 0.9981 \\
& AII & 0.5856 & 0.9989 \\
& AIII & 0.5424 & 0.9889 \\
& & $0.56 \pm 0.02$ & \\
\cline { 2 - 4 } & BI & 0.3360 & 0.9843 \\
& BII & 0.4032 & 0.9965 \\
& BIII & 0.3696 & 0.9944 \\
& & $0.37 \pm 0.03$ & \\
\hline \multirow{4}{*}{ PBR } & 1 & 0.7824 & 0.9954 \\
& 2 & 0.6720 & 0.9771 \\
& 3 & 0.6192 & 0.9656 \\
& & $0.69 \pm 0.08$ & \\
\cline { 2 - 4 } & 4 & 0.3384 & 0.9180 \\
& 5 & 0.4776 & 0.9312 \\
& & $0.41 \pm 0.10$ & \\
\hline
\end{tabular}

Table 1. The Gompertz specific growth rate of microalgae in seven experiments, with the corresponding correlation values. The mean value and the standard deviation are reported for the cultures with continuous illumination (Experiments A and B in the Roux bottles, and 1,2, and 3 in the PBR) and with light/dark cycles (Experiments 4 and 5 in the PBR). 


\subsection{Fluorescence measurements}

Variations in fluorescence intensity were successfully measured according to the increment in C. reinhardtii concentration. Some selected spectra are shown in Figure 3 for experiment A. The fluorescence dataset for experiments A and B are shown in suplementary material (File S1). In all cases, Chl fluorescence exhibits a peak around $\lambda=685 \mathrm{~nm}$ and a broad shoulder around $\lambda=740 \mathrm{~nm}$. This is a general observation at room temperature for Chl a (Gouveia-Neto et al., 2011; Krause and Weis, 1984). The fluorescence around $\lambda=685 \mathrm{~nm}$ is attributed to the $P S I I$ antenna, and the fluorescence around $\lambda=740 \mathrm{~nm}$ is due to the PSI antenna (Gouveia-Neto et al., 2011). The fluorescence signal/noise ratio in the measurements was around 7 at $\lambda=740 \mathrm{~nm}$ and 14 at $\lambda=685 \mathrm{~nm}$.

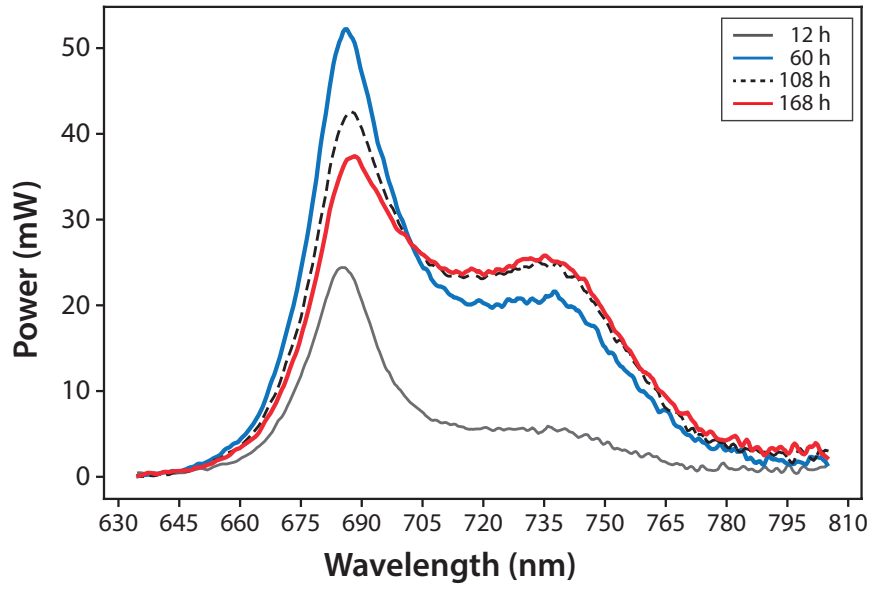

Figure 3. Microalgae fluorescence emission spectra ( $298 \pm 2 K)$ : evolution over seven days (168 h). Experiment $A, x_{0}=(34 \pm 2) \mathrm{mg} / \mathrm{L}$

In Figure 4, fluorescence evolution at $\lambda=685 \mathrm{~nm}$ and at $\lambda=740 \mathrm{~nm}$ is shown for all bottles of Experiments $\mathrm{A}$. The plots include trend lines. In both Experiments $\mathrm{A}$ and $\mathrm{B}$, the maximum fluorescence intensity at $\lambda=685 \mathrm{~nm}$ occurred between 48 and 60 hours. After that time, fluorescence decreased. Regarding fluorescence at $\lambda=740 \mathrm{~nm}$, in Experiment $\mathrm{A}$, the maximum value occurred at 72 hours in all cultures. In Experiment B, the maximum value happened between 96 and 108 hours. For both experiments, the maximum fluorescence at $\lambda=685 \mathrm{~nm}$ occurred before that of $\lambda=740 \mathrm{~nm}$. Maximum fluorescence at $\lambda=740 \mathrm{~nm}$ occurred at the highest algae concentrations. In general, the results were consistent with those described in the literature for fluorescence in plants (Gouveia-Neto et al., 2011). Namely, at low $C h l$ concentrations, fluorescence emissions increased with increasing $C h l$ concentration. At higher concentrations, the increase of fluorescence with the increment of $\mathrm{Chl}$ was detected only around $\lambda=740 \mathrm{~nm}$.

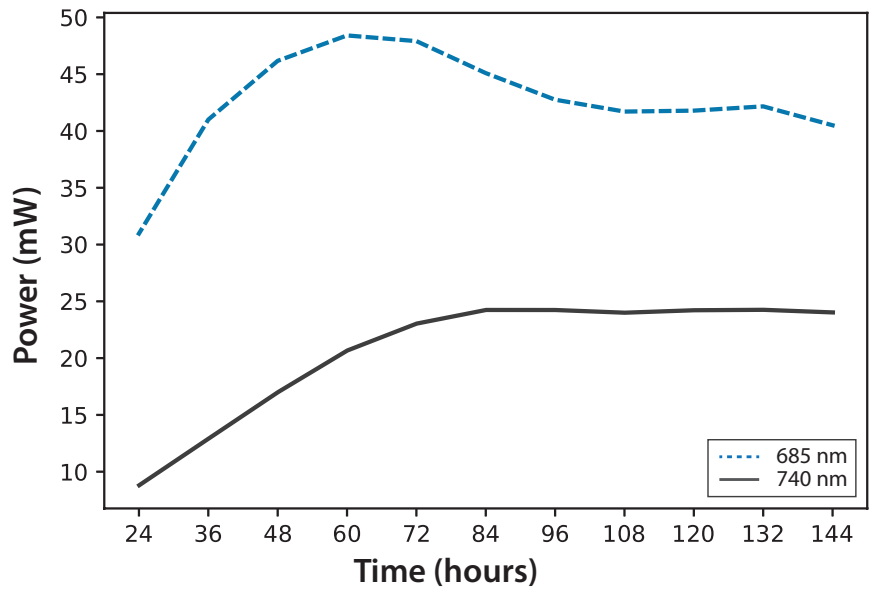

Figure 4. Experiment A: C. reinhardtii fluorescence evolution trend at $\lambda=685 \mathrm{~nm}$ and $\lambda=740 \mathrm{~nm}$ 
Regarding the $F_{685} / F_{740}$ ratio, we observed a similar behavior in every culture of both experiments, regardless of the initial concentration. For that reason, Figure 5 shows the average of the three cultures for each of the two experiments over five days. Over time, the $F_{685} / F_{740}$ fluorescence ratio decreased, meaning that the photosynthetic processes had improved. The same trend has been reported for green leaves in plants, as stated in equation 1 (Hák et al., 1990).

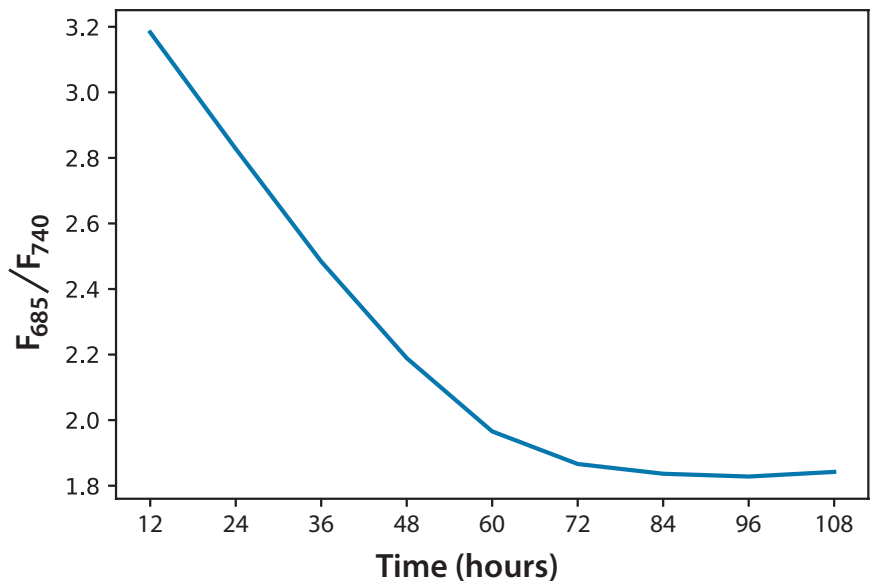

Figure 5. Fluorescence ratio $\left(F_{685} / F_{740}\right)$ trend for $C$. reinhardtii for all experiments.

Based on the information in Figure 5, and considering the data of 168 hours as the minimum possible value (aged cultures), we figured that cultures in both experiments reached around $70 \%$ maturity at 96 hours. Therefore, every culture evolved successfully and the conditions were appropriate to grow the microalgae and keep them in a good state of health. In addition, the cultures that reached the lowest $F_{685} / F_{740}$ values, that is, the highest photosynthetic activity, were those of Experiment A, which started with the lowest initial concentration. Moreover, after 72 hours, these values did not change significantly. This is the moment when illumination may not be enough for the culture because cell concentration reduces the passage of light. Equation 2 expresses a very useful linear correlation between the logarithmic concentration of microalgae and the $F_{685} / F_{740}$ ratio through time (Figure 6):

$$
\ln \left(\frac{x}{x_{0}}\right)=3.27-0.7084\left(F_{685} / F_{740}\right)
$$

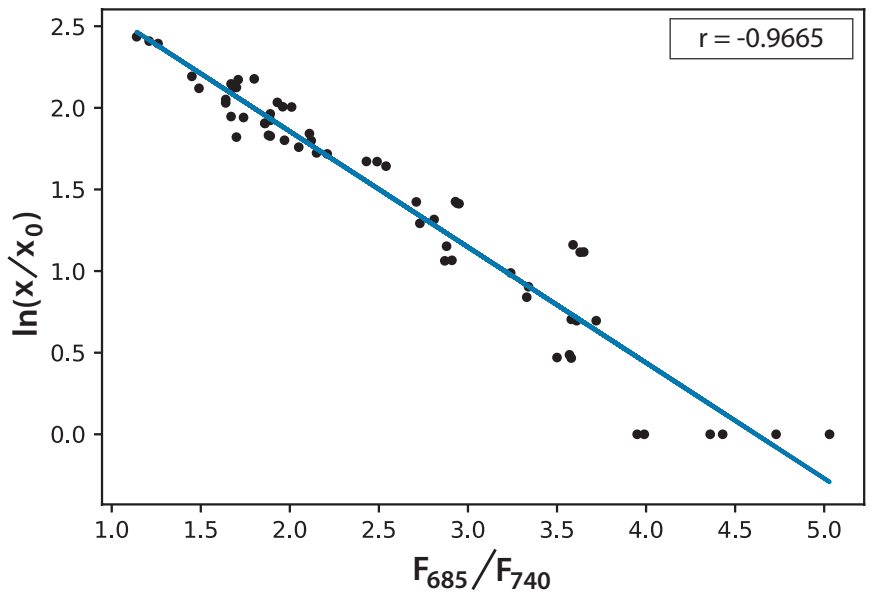

Figure 6. Linear correlation for the logarithmic microalgae concentration and the fluorescence ratio $F_{685} / F_{740}(r=-0.966578)$ 


\subsection{Digital images}

When the $P B R$ was used, digital images of the cultures were taken to monitor the change in color and to measure the penetration of a fluorescent beam during microalgae growth. Figure 7 shows a selection of images illustrating a typical experiment. We observed that cultures got darker with time due to the increase of biomass concentration in the $P B R$ preventing the passage of light throughout the reactor. For the fluorescence measurements, the flashes due to the blue super-luminescent diode were filtered in order to measure only fluorescence light contribution. This contribution diminishes with time due to a shadow effect produced by cells as the microalgae concentration gets denser.

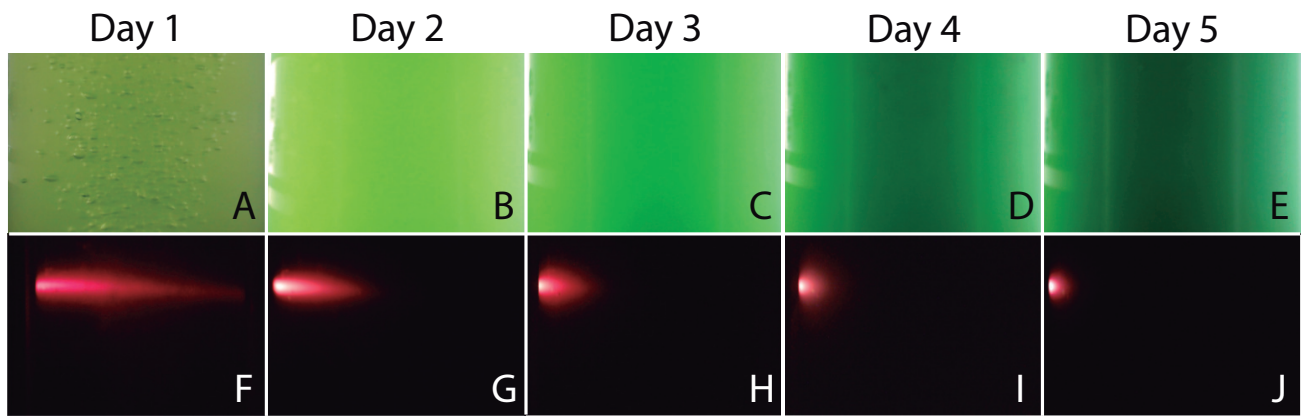

Figure 7. Representative images of the $P B R$ captured every day during an experiment. Color assessment (Figures 7A-7E) and fluorescent beam penetration (Figures 7F-7J).

CIELAB measurements include three values to characterize the color of a sample: L is the luminosity, the parameter "a" represents colors from green to red, and the parameter "b" represents colors from blue to yellow. Both parameters " $a$ " and " $b$ " remained almost constant throughout the experiment during microalgae growth. This means that, technically, color does not change. This is expected since the photosynthetic pigments are always the same and luminosity is what more importantly diminishes during cell growth since cells deflect or shadow light sources. Figure 8 shows the logarithmic correlation between microalgae concentration $\mathrm{x}$, and luminosity L for Experiments 1-3. For these experiments, equation 3 is proposed to get $x$ from on-line measurements of $\mathrm{L}$ from digital images:

$$
\ln \left(\frac{x}{x_{0}}\right)=(1.6 \pm 0.2)-(0.44 \pm 0.04)(L / W)
$$

In this equation, the intercept and the slope values are presented as the mean and the corresponding standard deviation of the individual correlations in the three experiments (see Figure 8). However, this same correlation was not observed in Experiments 4-5, where light/dark cycles were performed (Figure 9). The correlations between the values of the three experiments confirm that the experiments are reproducible, which gives confidence to the study. Table 2 shows the $L(W)$ and Table 3 shows the correlation between $\ln \left(x / x_{0}\right)$ values.

\begin{tabular}{cccc}
\hline & E1 & E2 & E3 \\
\hline E1 & 1.000000 & 0.963332 & 0.942350 \\
E2 & 0.963332 & 1.000000 & 0.973427 \\
E3 & 0.942350 & 0.973427 & 1.000000 \\
\hline
\end{tabular}

Table 2. $L(W)$ Correlation

Finally, the fluorescence beam penetration was characterized as an image changing with time. Both the beam surface area (data not shown) and penetration distance were used to monitor these changes. Similar results were obtained when comparing the cell concentration with the changes in the fluorescent images; therefore, only the distance beam penetration was used since its measurement was much simpler than that of the surface area. Figure 10 shows the correlation of this distance measured for the fluorescent beam penetration with the inverse of the OD. As stated before, the OD is related to the biomass concentration (del 


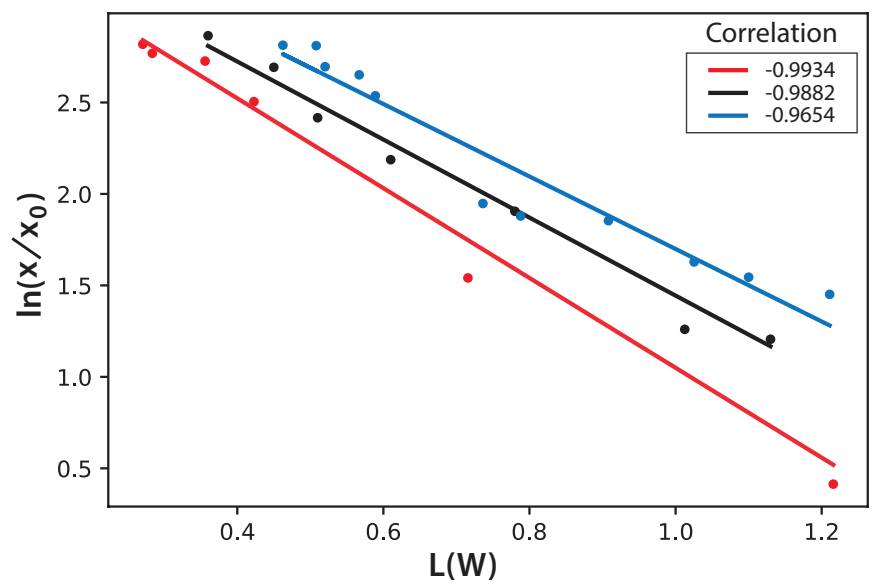

Figure 8. Logarithmic dependence of cell concentration with the luminosity (L) value in the CIELAB scale of colors for the PBR experiments with continuous illumination. The colors correspond to experimental values: black (exp. 1), red (exp. 2), and blue (exp. 3); the lines correspond to the mean-squares correlations

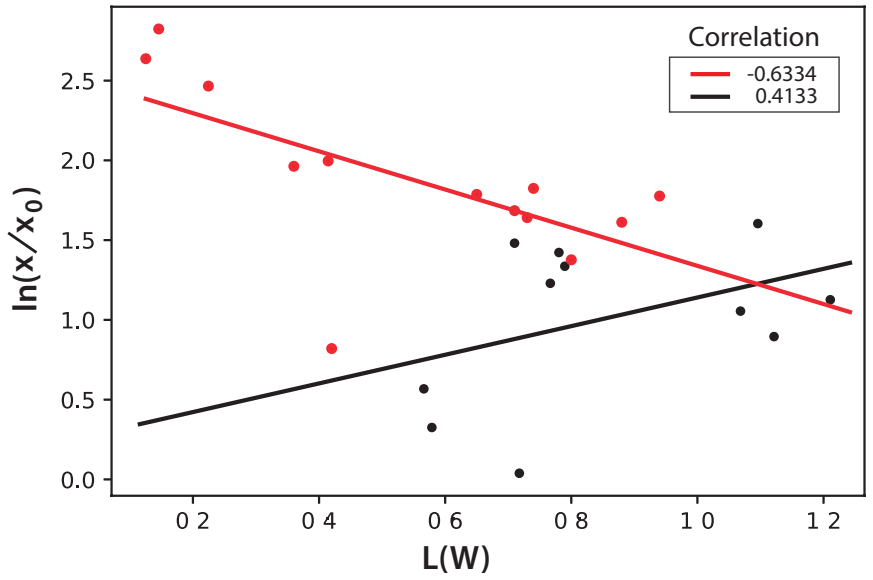

Figure 9. Logarithmic dependence of cell concentration with the luminosity (L) value in the CIELAB scale of colors for the PBR experiments with light/dark cycles. The colors correspond to experimental values: black (exp. 4), red (exp. 5); the lines correspond to the mean-squares correlations

\begin{tabular}{cccc}
\hline & E1 & E2 & E3 \\
\hline E1 & 1.000000 & 0.973283 & 0.948333 \\
E2 & 0.973283 & 1.000000 & 0.985426 \\
E3 & 0.948333 & 0.985426 & 1.000000 \\
\hline
\end{tabular}

Table 3. $\ln \left(x / x_{0}\right)$ Correlation

Campo et al., 2014). It is important to note that the linear correlations were obtained for all experiments, with equation 4 proposed to calculate the OD of the culture directly from the on-line measure of the beam penetration:

$$
O D=\frac{1}{\text { beam penetration } / \mathrm{cm}}
$$

This simple equation is proposed since the values for the mean and the corresponding standard deviation for the intercept and the slope in the individual linear correlations for the five experiments are 
$(0.0 \pm 0.5)$ and $(1.0 \pm 0.1) \mathrm{cm}^{-1}$ respectively.

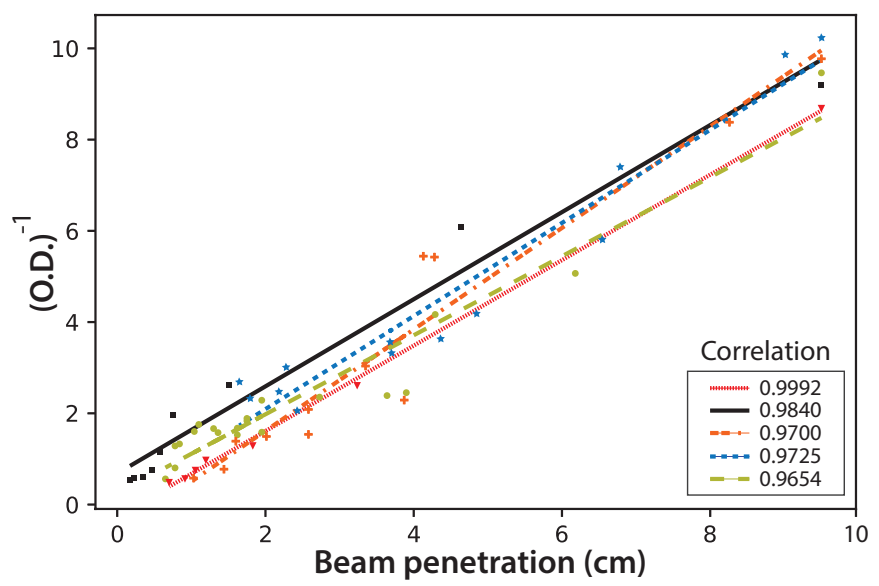

Figure 10. The inverse of microalgae culture optical density (OD) as a function of the fluorescent beam penetration in the PBR. The colors correspond to the following experimental values: red dash line (exp. 1), black (exp. 2), orange dotted line (exp. 3), blue dotted line (exp. 4) and light green dotted line (exp. 5); the lines correspond to the least-squares correlations.

\section{CONCLUSIONS}

The growth of $C$. reinhardtii cultures was successfully monitored through off-line and on-line optical techniques at an affordable cost. It was confirmed that, as evidenced in green plants, the maximum fluorescence around $\lambda=685 \mathrm{~nm}$ occurs before that at $\lambda=740 \mathrm{~nm}$. The maximum fluorescence at $\lambda=740$ $\mathrm{nm}$ occurs at a higher concentration, than what is needed at $\lambda=685 \mathrm{~nm}$. Once the maximum fluorescence at $\lambda=685 \mathrm{~nm}$ has been reached, it decreases earlier and at a faster rate than the fluorescence at $\lambda=740 \mathrm{~nm}$. Although the $F_{685} / F_{740}$ fluorescence ratio is a well-known method, it was used for $C$. reinhardtii cultures for the first time here. A very useful linear correlation occurs between the logarithmic concentration of $C$. reinhardtii and the $F_{685} / F_{740}$ ratio over time.

Moreover, the on-line analysis of digital images was shown to be useful in monitoring $C$. reinhardtii growth. The luminosity measurements in the CIELAB scale correlated linearly with the microbial concentration for cultures under continuous illumination. However, this correlation was not found for the cultures in a light/dark regime. Nevertheless, both the fluorescent beam penetration images' distance and the surface captured for the beam linearly correlated with optical density and, consequently, with the microalgae culture density for all the illumination regimes. A simple reciprocal equation can be used to calculate optical density as the inverse of the measured distance of beam penetration $(\lambda=440 \mathrm{~nm})$.

The on-line techniques proposed here are very practical for both research and the study of industrial microalgae cultures. In the case of multispectral remote sensing reflectances at both $685 \mathrm{~nm}$ and 740 $\mathrm{nm}$, and with the contribution of field measurements for calibration, a future study should use equation 2 and regression models between the logarithmic concentration of microalgae and remote sensing data to estimate the concentration of Chlorophyll a in wide water areas.

\section{ACKNOWLEDGMENTS}

Hugo Lazcano thanks CONACyT for his postdoctoral fellowship and for the support provided through the "Cátedras-CONACYT" program (project 526). The authors are grateful to the editor and reviewers for improving this manuscript. We thank Moisés Perales for his help with editing.

\section{REFERENCES}

Abdel-Raouf, N., Al-Homaidan, A., and Ibraheem, I. (2012). Microalgae and wastewater treatment. Saudi journal of biological sciences, 19(3):257-275. 
Allen, J. F. (1992). How does protein phosphorylation regulate photosynthesis? Trends in biochemical sciences, 17(1):12-17.

Antal, T., Konyukhov, I., Volgusheva, A., Plyusnina, T., Khruschev, S., Kukarskikh, G., Goryachev, S., and Rubin, A. (2019). Chlorophyll fluorescence induction and relaxation system for the continuous monitoring of photosynthetic capacity in photobioreactors. Physiologia plantarum, 165(3):476-486.

Breijo, F. J. G., Caselles, J. R., and Siurana, P. S. (2006). Introducción al funcionamiento de las plantas. Ed. Univ. Politéc. Valencia.

Carvalho, A. P., Meireles, L. A., and Malcata, F. X. (2006). Microalgal reactors: a review of enclosed system designs and performances. Biotechnology progress, 22(6):1490-1506.

Castorina, P., Delsanto, P., and Guiot, C. (2006). Classification scheme for phenomenological universalities in growth problems in physics and other sciences. Physical review letters, 96(18):188701.

Chekalyuk, A. and Hafez, M. (2008). Advanced laser fluorometry of natural aquatic environments. Limnology and Oceanography: Methods, 6(11):591-609.

del Campo, J. S. M., Escalante, R., Robledo, D., and Patino, R. (2014). Hydrogen production by chlamydomonas reinhardtii under light-driven and sulfur-deprived conditions: using biomass grown in outdoor photobioreactors at the yucatan peninsula. international journal of hydrogen energy, 39(36):20950-20957.

Gorbunov, M. Y. and Falkowski, P. G. (2004). Fluorescence induction and relaxation (fire) technique and instrumentation for monitoring photosynthetic processes and primary production in aquatic ecosystems. In Photosynthesis: Fundamental Aspects to Global Perspectives"-Proc. 13th International Congress of Photosynthesis, Montreal, Aug, pages 1029-1031.

Gouveia-Neto, A. S., da Silva-Jr, E. A., Cunha, P. C., Oliveira-Filho, R. A., Silva, L. M., da Costa, E. B., Camara, T. J., and Willadino, L. G. (2011). Abiotic stress diagnosis via laser induced chlorophyll fluorescence analysis in plants for biofuel. In Biofuel Production-Recent Developments and Prospects. IntechOpen.

Griffiths, M. J., Garcin, C., van Hille, R. P., and Harrison, S. T. (2011). Interference by pigment in the estimation of microalgal biomass concentration by optical density. Journal of microbiological methods, 85(2):119-123.

Gupta, P. L., Lee, S.-M., and Choi, H.-J. (2015). A mini review: photobioreactors for large scale algal cultivation. World Journal of Microbiology and Biotechnology, 31(9):1409-1417.

Hák, R., Lichtenthaler, H., and Rinderle, U. (1990). Decrease of the chlorophyll fluorescence ratio f690/f730 during greening and development of leaves. Radiation and environmental biophysics, 29(4):329-336.

Havlik, I., Scheper, T., and Reardon, K. F. (2016). Monitoring of Microalgal Processes, pages 89-142. Springer International Publishing, Cham.

Johnson, Z. I. (2004). Description and application of the background irradiance gradient-single turnover fluorometer (big-stf). Marine Ecology Progress Series, 283:73-80.

Kolber, Z. S., Prášil, O., and Falkowski, P. G. (1998). Measurements of variable chlorophyll fluorescence using fast repetition rate techniques: defining methodology and experimental protocols. Biochimica et Biophysica Acta (BBA)-Bioenergetics, 1367(1-3):88-106.

Krause, G. and Weis, E. (1991). Chlorophyll fluorescence and photosynthesis: the basics. Annual review of plant biology, 42(1):313-349.

Krause, G. H. and Weis, E. (1984). Chlorophyll fluorescence as a tool in plant physiology. Photosynthesis research, 5(2):139-157.

Kromkamp, J. C., Beardall, J., Sukenik, A., Kopeckỳ, J., Masojídek, J., Van Bergeijk, S., Gabai, S., Shaham, E., and Yamshon, A. (2009). Short-term variations in photosynthetic parameters of nannochloropsis cultures grown in two types of outdoor mass cultivation systems. Aquatic Microbial Ecology, 56(2-3):309-322.

Masojídek, J., Kopeckỳ, J., Giannelli, L., and Torzillo, G. (2011). Productivity correlated to photobiochemical performance of chlorella mass cultures grown outdoors in thin-layer cascades. Journal of industrial microbiology \& biotechnology, 38(2):307-317.

Masojídek, J., Vonshak, A., and Torzillo, G. (2010). Chlorophyll fluorescence applications in microalgal mass cultures. In Chlorophyll a fluorescence in aquatic sciences: methods and applications, pages 277-292. Springer.

Mata, T. M., Martins, A. A., and Caetano, N. S. (2010). Microalgae for biodiesel production and other 
applications: a review. Renewable and sustainable energy reviews, 14(1):217-232.

Mauzerall, D. (1972). Light-induced fluorescence changes in chlorella, and the primary photoreactions for the production of oxygen. Proceedings of the National Academy of Sciences, 69(6):1358-1362.

Maxwell, K. and Johnson, G. N. (2000). Chlorophyll fluorescence-a practical guide. Journal of experimental botany, 51(345):659-668.

Misra, A. N., Misra, M., and Singh, R. (2012). Chlorophyll fluorescence in plant biology. In Biophysics. IntechOpen.

Niccolai, A., Zittelli, G. C., Rodolfi, L., Biondi, N., and Tredici, M. R. (2019). Microalgae of interest as food source: Biochemical composition and digestibility. Algal Research, 42:101617.

Olson, R. J., Chekalyuk, A. M., and Sosik, H. M. (1996). Phytoplankton photosynethetic characteristics from fluorescence induction assays of individual cells. Limnology and oceanography, 41(6):1253-1263.

Pulz, O. (2001). Photobioreactors: production systems for phototrophic microorganisms. Applied microbiology and biotechnology, 57(3):287-293.

Pulz, O. and Gross, W. (2004). Valuable products from biotechnology of microalgae. Applied microbiology and biotechnology, 65(6):635-648.

Roháček, K., Soukupová, J., and Barták, M. (2008). Chlorophyll fluorescence: a wonderful tool to study plant physiology and plant stress. Plant Cell Compartments-Selected Topics. Research Signpost, Kerala, India, pages 41-104.

Rym, B. D. (2012). Photosynthetic behavior of microalgae in response to environmental factors. In Applied Photosynthesis. IntechOpen.

Show, K.-Y., Yan, Y., Zong, C., Guo, N., Chang, J.-S., and Lee, D.-J. (2019). State of the art and challenges of biohydrogen from microalgae. Bioresource technology, page 121747.

Spolaore, P., Joannis-Cassan, C., Duran, E., and Isambert, A. (2006). Commercial applications of microalgae. Journal of bioscience and bioengineering, 101(2):87-96.

Sueoka, N. (1960). Mitotic replication of deoxyribonucleic acid in chlamydomonas reinhardi. Proceedings of the National Academy of Sciences of the United States of America, 46(1):83.

Sukenik, A., Beardall, J., Kromkamp, J. C., Kopeckỳ, J., Masojídek, J., van Bergeijk, S., Gabai, S., Shaham, E., and Yamshon, A. (2009). Photosynthetic performance of outdoor nannochloropsis mass cultures under a wide range of environmental conditions. Aquatic Microbial Ecology, 56(2-3):297-308.

Torzillo, G., Scoma, A., Faraloni, C., and Giannelli, L. (2015). Advances in the biotechnology of hydrogen production with the microalga chlamydomonas reinhardtii. Critical reviews in biotechnology, 35(4):485-496.

Wang, B., Li, Y., Wu, N., and Lan, C. Q. (2008). Co 2 bio-mitigation using microalgae. Applied microbiology and biotechnology, 79(5):707-718.

Xu, L., Weathers, P. J., Xiong, X.-R., and Liu, C.-Z. (2009). Microalgal bioreactors: challenges and opportunities. Engineering in Life Sciences, 9(3):178-189. 